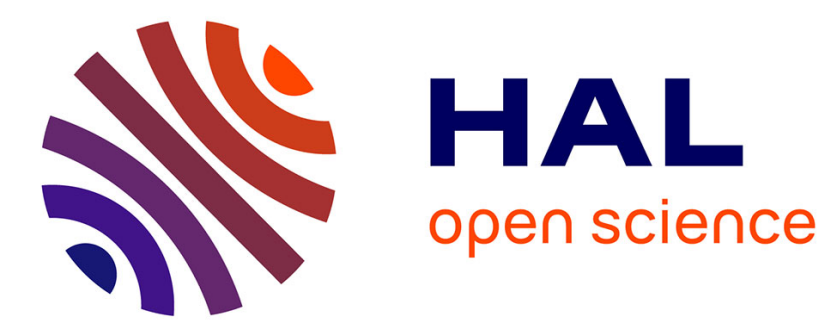

\title{
Unsupervised segmentation based on Von Mises circular distributions for orientation estimation in textured images
}

Jean-Pierre da Costa, Frédéric Galland, Antoine Roueff, Christian Germain

\section{- To cite this version:}

Jean-Pierre da Costa, Frédéric Galland, Antoine Roueff, Christian Germain. Unsupervised segmentation based on Von Mises circular distributions for orientation estimation in textured images. Journal of Electronic Imaging, 2012, 21 (2), pp.021102. 10.1117/1.JEI.21.2.021102 . hal-00700943

\section{HAL Id: hal-00700943 \\ https://hal.science/hal-00700943}

Submitted on 24 May 2012

HAL is a multi-disciplinary open access archive for the deposit and dissemination of scientific research documents, whether they are published or not. The documents may come from teaching and research institutions in France or abroad, or from public or private research centers.
L'archive ouverte pluridisciplinaire HAL, est destinée au dépôt et à la diffusion de documents scientifiques de niveau recherche, publiés ou non, émanant des établissements d'enseignement et de recherche français ou étrangers, des laboratoires publics ou privés. 


\title{
Unsupervised segmentation based on Von Mises circular distributions for orientation estimation in textured images
}

\author{
Jean-Pierre Da Costa ${ }^{a}$, Frédéric Galland ${ }^{b}$, Antoine Roueff $^{b}$, Christian Germain $^{a}$ \\ ${ }^{a}$ IMS Laboratory, UMR5218 CNRS, Université de Bordeaux, 351 Cours de la Libération, \\ 33405 Talence cedex, France \\ ${ }^{b}$ Institut Fresnel, CNRS, Aix-Marseille Université, Ecole Centrale Marseille, Campus de \\ Saint-Jérôme, 13013 Marseille, France
}

\begin{abstract}
In this paper, which deals with textured images and more particularly with directional textures, a new parametric technique is proposed to estimate the orientation field of textures. It consists of segmenting the image into regions with homogeneous orientations, and estimating the orientation inside each of these regions. This allows us to maximize the size of the samples used to estimate the orientation without being corrupted by the presence of boundaries between regions. For that purpose, the local - hence noisy - orientations of the texture are first estimated using small filters $(3 \times 3$ pixels $)$. The segmentation of the obtained orientation field image then relies on a generalization of a Minimum Description Length (MDL) based segmentation technique, to the case of $\pi$ periodic circular data modeled with Von Mises probability density functions. This leads to a fast segmentation algorithm without tuning parameters in the optimized criterion. The accuracy of the orientations estimated with the proposed method is then compared with other approaches on synthetic images and an application to the processing of real images is finally addressed.
\end{abstract}

Keywords: Texture, orientation, circular data, Von Mises, segmentation, polygonal active grid, minimum description length

\section{INTRODUCTION}

Texture is a keystone of many image analysis frameworks. Whether for classification, for indexation or for segmentation applications, the importance of texture descriptors cannot be overlooked when dealing with natural images. Among such textural descriptors, pattern orientation has proved to be crucial for human image perception 112 Besides, machine vision systems have also benefited from orientation and anisotropy-based features in many domains (e.g. $\cdot 3-5)$.

Contact: jean-pierre.dacosta@ims-bordeaux.fr, frederic.galland@fresnel.fr 
In particular, some papers have reported the use of pattern orientations and texture anisotropies for segmentation purposes. To mention a few, Bigün and Du Buf ${ }^{6}$ used complex moments in Gabor space to capture the symmetries of the local image content within a quad tree segmentation approach. Rousson et al.7 and Luis García et al. ${ }^{8}$ inspired by tensor-valued image analysis techniques, used the structure tensor ${ }^{9}[10$ as a textural feature driving level-sets segmentation frameworks. Recently, the same authors proposed a combined segmentation approach using both tensor and color information within a common minimization framework $\frac{11}{11}$ In prior works, Da Costa et al ${ }^{12}[13$ have also taken advantage of texture anisotropy for the segmentation of remote-sensed images. Anisotropy was captured either by spectral Gabor features or by spatial anisotropy descriptors, and was used either in a region growing segmentation framework ${ }^{[12}$ or simply in a thresholding/regularizing scheme ${ }^{[13}$ In the context of manuscript analysis, Grana et al ${ }^{14}$ proposed a classification algorithm to discriminate between sub blocks of text, illustrations and background using anisotropy. Orientation distributions inside sub blocks were captured by grey level autocorrelation and modelled using mixtures of Von Mises density functions $\frac{15}{15}$

In this paper, segmentation is not considered as an aim of image analysis but as a means of estimating orientations in textures. Our contribution is a segmentation-based parametric approach for the estimation of orientations in directional textures, i.e. textures exclusively composed of oriented patterns 16$] 17$ The approach relies on a first step of local estimation of the orientations in the image using derivative techniques $\frac{16}{16}$ The obtained orientation field image is segmented into homogeneous regions, using the Minimum Description Length (MDL) segmentation technique formerly presented in $\frac{18}{18}$ and generalized in this paper to $\pi$-periodic data modeled with circular Von Mises distributions. ${ }^{15}$ A final orientation estimation is performed by averaging low level orientations inside the segmented regions.

The paper is organized as follows. Section 2 is a methodological presentation dealing with orientation estimation and circular data modelling. Section 3 presents a generalization of the MDL segmentation technique to Von Mises circular data. Section 4 reports an experimental evaluation of the segmentation-based framework for orientation estimation on synthetic and real textured images. The last section provides some conclusions.

\section{ORIENTATION AND TEXTURES}

\subsection{Textures of interest}

As orientation relates to visual perception, defining the concept of image orientation is not trivial. The notion of orientation is often closely linked to the kind of patterns observed in the images. In this paper, only the case where a unique orientation (i.e. the orientation of the underlying oriented pattern) is present at any pixel of the image will be considered. This corresponds to the linearly symmetric model, $\frac{10}{10}$ to the $\{i 1 D\}$ case - i.e. 
intrinsic dimensionality is 1 - according to Krieger and Zetzsche's nomenclature ${ }^{\sqrt{19}}$ or to the mono-directional texture case mentioned by Michelet et al ${ }^{17}$ The limitation to unique orientations excludes some textures where superimpositions or occlusions occur, ${ }^{20}$ but such textures are beyond the scope of this paper.

\subsection{Orientation Estimation}

It is commonly accepted that anisotropy perception is closely linked to the scale at which an image is considered. The multi-scale nature of orientation information has therefore been taken into account in many vision systems both in spectral approaches $s\left[10\right.$ and in spatial approaches ${ }^{5}$ which usually attempt to capture anisotropy properties at various scales. However, in this paper we chose to focus on local orientations only, thus restricting orientation estimation to local pixel neighborhoods. The objective is twofold. First, keeping orientation estimates local limits border effects at the boundaries of regions with different orientations. Secondly, as local estimates can be processed by spatial filters operating within very small pixel neighborhoods, strong dependences between orientation estimates on neighboring pixels are avoided.

In the case of mono-directional textures, the use of local derivatives ${ }^{21}[22$ is a natural approach for orientation estimation. In this paper we use the formerly proposed ${ }^{\sqrt{16}}$ gradient operator GOP. Similar to standard $3 \times 3$ operators (e.g. Sobel filter), GOP is part of a panel of filters designed to limit both bias and noise sensitivity while keeping the estimation process as local as possible. Local texture orientation is orthogonal to the orientation of the gradient. It is defined within an interval of width $\pi$, for instance ] $\left.-\frac{\pi}{2}, \frac{\pi}{2}\right]$, as two values that are $\pi$ apart refer to the same orientation. Such orientation data are called axial dat $a^{15}$ i.e. $\pi$-periodic circular data.

Nevertheless, in the case of noisy images, orientation estimations are very scattered and cannot be used directly. For example, the estimation of orientations inside the textured image of fig. 1. a is shown on fig. 1. b, using the GOP operator. In order to reduce the noise in these images, a classical solution consists in averaging the orientations in a small neighborhood $V(x, y)$ around $(x, y)$. In this paper, $V(x, y)$ is defined as a sliding square window containing $N_{V}=d \times d$ pixels and centered on pixel $(x, y)$. Let $\theta(x, y)$ be the orientation estimated at pixel $(x, y)$. Thus the double angle $\alpha(x, y)=2 \theta(x, y)$ is a $2 \pi$-periodic variable. Its averaging $\bar{\alpha}$ can be computed inside $V(x, y)$ as suggested by Mardia and Jupp! $\frac{15}{15}$

$$
2 \bar{\theta}(x, y)=\bar{\alpha}(x, y)=\tan ^{-1} \frac{\bar{S}(x, y)}{\bar{C}(x, y)}
$$

where

$$
\left\{\begin{array}{l}
\bar{C}(x, y)=\frac{1}{N_{V}} \sum_{(u, v) \in V(x, y)} \cos \alpha(u, v) \\
\bar{S}(x, y)=\frac{1}{N_{V}} \sum_{(u, v) \in V(x, y)} \sin \alpha(u, v)
\end{array}\right.
$$


with $N_{V}$ the number of pixels in $V(x, y)$. In equations 112 angular values are replaced by unit vectors $(\cos \alpha(u, v), \sin \alpha(u, v))$ which are averaged within $V(x, y)$. This avoids erroneous estimations provided by classical averaging when angular data close to the limits of the orientation interval occur ( $-\pi$ or $\pi$ for instance). The resulting orientation provided by this averaging technique (denoted AGOP in the following for Averaged GOP operator) is closely related to the orientation of the first eigen vector of the structure tensor ${ }^{9,10}$ which is another classical approach to get a regularized orientation estimation.

The averaging $\bar{\theta}(x, y)=\bar{\alpha}(x, y) / 2$ of the orientation image of fig. 1. b obtained using squared sliding windows $V(x, y)$ with different side sizes $d$ (and thus $N_{V}=d \times d$ pixels) are reported on fig. 11. $(d=7)$ and fig. 1 1 d $(d=11)$. It is notably shown on the left part of fig. 3 that the Mean Average Deviation (MAD) between the averaged estimated orientation $\{\bar{\theta}(x, y)\}_{(x, y)}$ and the true orientation $\left\{\theta_{G T}(x, y)\right\}_{(x, y)}$ (GT for Ground Truth) used to generate the data (see fig. 1 1 h), is minimal for $d=7$, using the following definition for the MAD:

$$
M A D=\frac{1}{N} \sum_{(x, y) \in \text { Image }} \min \left(\left|\theta_{G T}(x, y)-\bar{\theta}(x, y)\right|, \pi-\left|\theta_{G T}(x, y)-\bar{\theta}(x, y)\right|\right)
$$

where $N$ is the number of pixels in the image. Of course, the value $d$ which minimizes the MAD, denoted $\widetilde{d}^{M A D}$

in the following, highly depends on the image considered and on the noise level. For instance, $\widetilde{d}^{M A D}=11$ when the noise level is increased by a factor $2(\sigma=100)$ as shown in fig. 2 .

In real applications, where the ground truth is unknown and thus quality criteria such as the MAD cannot be calculated, the estimation of the size of this sliding window is a difficult problem. In this paper, instead of trying to find the best window size, it is proposed to directly recover the different homogeneous regions in the image by decomposing the orientation field into a patchwork of regions with homogeneous orientations. Inside each homogeneous region, the whole pixels can then be used to refine the orientation estimation. The estimation of the orientation is thus averaged on the biggest homogeneous sample of pixels, but without being corrupted by the presence of inhomogeneities near borders.

\section{PARTITION INTO REGIONS WITH HOMOGENEOUS ORIENTATIONS}

Let $\theta=\left\{\theta(x, y),(x, y) \in\left[1, N_{x}\right] \times\left[1, N_{y}\right]\right\}$ be an image of orientations composed of $N=N_{x} \times N_{y}$ pixels. This image is assumed to be composed of a patchwork of $R$ homogeneous regions $\Omega_{r}(r \in[1, R])$. In other words, the pixel orientations in each region $\Omega_{r}$ are assumed to be independent realizations of a probability density function (PDF) $P_{\left[\beta_{r}\right]}$, where $\beta_{r}$ denotes the parameter vector of the PDF. A partition is defined with the rule $(x, y) \in \Omega_{r} \Longrightarrow \theta(x, y) \sim P_{\left[\beta_{r}\right]}$. The goal of image partitioning is then to retrieve the underlying partition $w(x, y)$, so that $w(x, y)=r \Longleftrightarrow(x, y) \in \Omega_{r}$. The main difficulty of such a task is that the PDF $P_{\left[\beta_{r}\right]}, r \in[1, R]$ are unknown. 


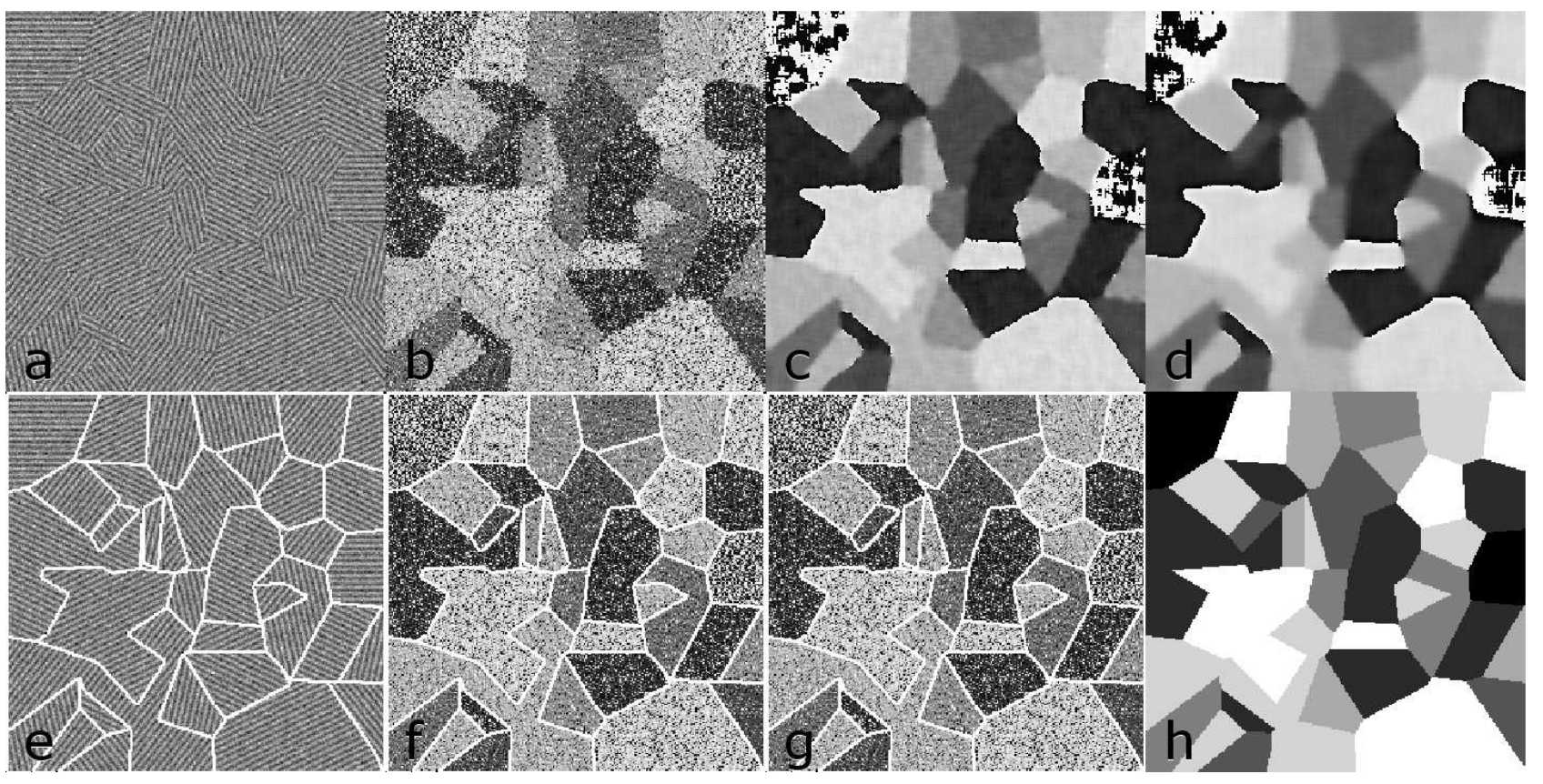

Figure 1. (a) Textured image obtained by adding to a non noisy image (with grey levels between 0 and 100) a zero-mean Gaussian noise with standard deviation $\sigma=50$. The orientation estimated using a $3 \times 3$ GOP estimator is shown on (b) and the smoothing of this orientation with a $d \times d$ sliding windows is shown for $d=7$ (c) and $d=11$ (d). The segmentation into homogeneous regions obtained with the proposed technique is shown on (e,f) (either displayed on (a) or (b)) and the associated estimated orientations are shown on (g). The orientation ground truth is shown on (h). In this case $\widetilde{d}^{M A D}=7$.

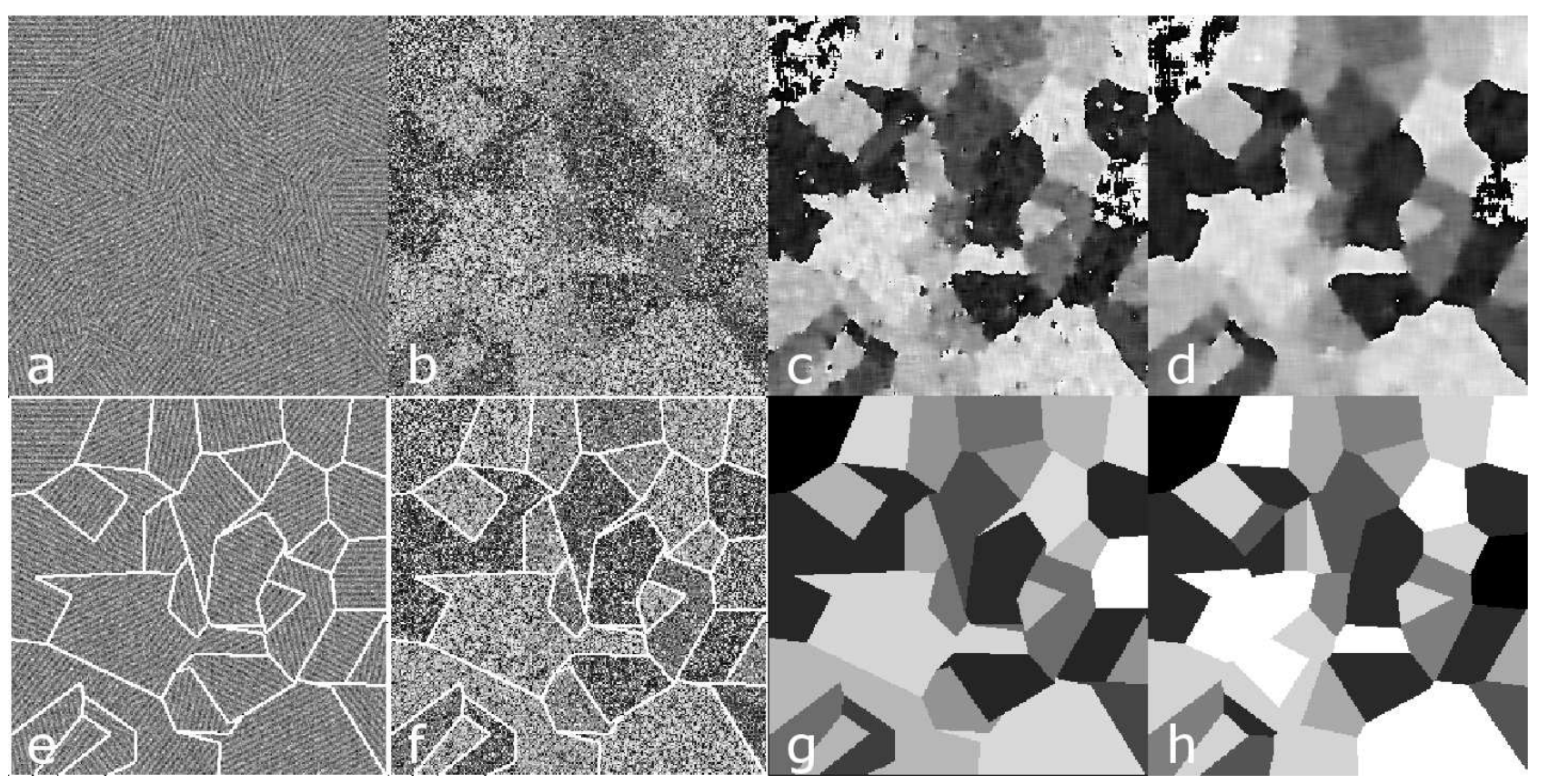

Figure 2. Same as figure 1 but with $\sigma=100$. In this case $\widetilde{d}^{M A D}=11$. 


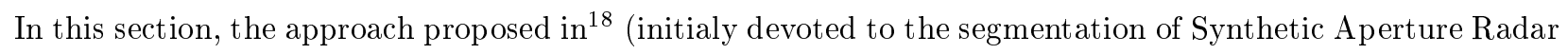
(SAR) images) is generalized to the segmentation of orientation field images. It relies on the minimization of a MDL criterion,,$\sqrt[23]{24}$ and it has been demonstrated $\sqrt{18} \mid 25$ that in the case of Gaussian, Gamma, Poisson or Bernoulli PDFs, it can lead to efficient techniques to obtain a decomposition of the image into statistically homogeneous regions. In the present case of orientation images, such approaches have to be extended to circular data.

\subsection{The Von Mises PDF for circular data}

Among all the PDF proposed in the literature to model $2 \pi$-periodic circular data, ${ }^{26}$ a lot of interest has been devoted to the Von Mises PDF,, 15 which has also been used for image processing to model orientation data in textures $\frac{14,[27}{27}$ This PDF is unimodal and symmetric and is entirely defined by two parameters: its mean orientation and its concentration. In this paper, the $\pi$-periodic PDF of the orientation $\theta(x, y)$ will be directly derived from this model, by considering that $\alpha(x, y)=2 \theta(x, y)$ is distributed according to the standard $2 \pi$ periodic Von Mises PDF, leading to

$$
P_{\left[\mu_{r}, \kappa_{r}\right]}(\alpha)=\frac{1}{2 \pi I_{0}\left(\kappa_{r}\right)} e^{\kappa_{r} \cos \left(\alpha-\mu_{r}\right)} .
$$

where $I_{0}$ is the modified Bessel function of the first kind and order 0 , and where $\left.\left.\mu_{r} \in\right]-\pi, \pi\right]$ and $\kappa_{r} \geq 0$ are respectively the mean orientation and the concentration of $\alpha$ inside $\Omega_{r}$. The mean orientation of $\theta=\alpha / 2$ is thus equal to $\mu_{r} / 2$ (modulo $\pi$ ).

In the following, the purpose will thus be to generalize the MDL-based partitioning technique proposed in ${ }^{18}[25$ to such circular data.

\subsection{Determination of the MDL criterion adapted to Von Mises data}

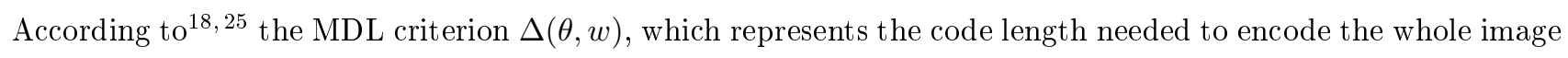
using the partition $w$, is the sum of three terms:

$$
\Delta(\theta, w)=\Delta_{G}(w)+\Delta_{P}(\beta \mid w)+\Delta_{L}(\theta \mid w, \beta)
$$

where $\Delta_{G}$ is the code length needed to encode the partition $w, \Delta_{P}$ is the code length needed to encode the PDF parameters $\beta_{r}$ in each region $\Omega_{r}$ defined by $w$, and $\Delta_{L}$ is the code length needed to encode the pixel orientation $\theta(x, y)$ inside each region $\Omega_{r}$ knowing $\beta=\left\{\beta_{r}\right\}_{r \in[1, R]}$. Let us now detail the expression of these three terms, as a function of the partition $w$.

Following ${ }^{18}$ it is proposed to model the partition $w$ with a polygonal grid, i.e. a set of nodes linked by segments to define the different regions $\Omega_{r}$. In this case, the code length $\Delta_{G}$ needed to encode a given partition 
$w$ can be approximated with $\frac{18}{18}$

$$
\Delta_{G}(w)=n(\log N+\log p)+\log p+2 p+p \log \left(4 \bar{d}_{x} \bar{d}_{y}\right)
$$

where $p$ is the number of segments of the polygonal grid, $n$ the minimum number of Eulerian graphs needed to describe the grid, and $\bar{d}_{x}$ and $\bar{d}_{y}$ the mean lengths of the horizontal and vertical projections of the grid segments and $N$ the number of pixels of the image (see ${ }^{\sqrt{18}}$ and ${ }^{\sqrt{28}}$ for details).

The code length needed to encode the PDF parameters $\beta_{r}$ inside each region can be approximated with!24

$$
\Delta_{P}(\beta \mid w)=\sum_{r=1}^{R} \frac{\operatorname{card}\left(\beta_{r}\right)}{2} \log \left(N_{r}\right)
$$

where $N_{r}$ is the number of pixels inside region $\Omega_{r}$ and where $\operatorname{card}\left(\beta_{r}\right)$ is the size of the parameter vector $\beta_{r}$, i.e. the number of PDF parameters that need to be encoded. In the present case, the parameter vector of the Von Mises PDF is $\beta_{r}=\left(\mu_{r}, \kappa_{r}\right)$, and thus $\operatorname{card}\left(\beta_{r}\right)=2$.

The last term $\Delta_{L}$ represents the code length needed to describe the pixel orientation $\theta(x, y)$ inside each region using an entropic code: 29

$$
\Delta_{L}(\theta \mid w, \beta)=\sum_{r=1}^{R}-\mathcal{L}\left(\Omega_{r} \mid \beta_{r}\right)
$$

where $\mathcal{L}\left(\Omega_{r} \mid \beta_{r}\right)$ is the log-likelihood of the sample $\left\{\alpha(x, y),(x, y) \in \Omega_{r}\right\}$, knowing the PDF parameter $\beta_{r}$. In the case of Von Mises PDF, one directly obtains! $\frac{15}{15}$

$$
\mathcal{L}\left(\Omega_{r} \mid \beta_{r}\right)=N_{r}\left(\log 2 \pi+\kappa_{r} \bar{R}_{r} \cos \left(\bar{\alpha}_{r}-\mu_{r}\right)-\log I_{0}\left(\kappa_{r}\right)\right)
$$

where

$$
\begin{aligned}
& \bar{\alpha}_{r}=\tan ^{-1} \frac{\bar{S}_{r}}{\bar{C}_{r}} \\
& \bar{R}_{r}=\sqrt{{\overline{C_{r}}}^{2}+\bar{S}_{r}{ }^{2}}
\end{aligned}
$$

and

$$
\left\{\begin{array}{l}
\bar{C}_{r}=\frac{1}{N} \sum_{(x, y) \in \Omega_{r}} \cos \alpha(x, y) \\
\bar{S}_{r}=\frac{1}{N} \sum_{(x, y) \in \Omega_{r}} \sin \alpha(x, y)
\end{array}\right.
$$

Since $\beta_{r}=\left(\mu_{r}, \kappa_{r}\right)$ is generally unknown, it has to be estimated. Following $\frac{15}{15}$ the parameters $\mu_{r}$ and $\kappa_{r}$ of the Von Mises PDF can be estimated in the Maximum Likelihood (ML) sense, leading to the following ML estimates $\widehat{\mu}_{r}$ and $\widehat{\kappa}_{r}$ :

$$
\begin{aligned}
& \widehat{\mu}_{r}=\bar{\alpha}_{r} \\
& \widehat{\kappa}_{r}=A^{-1}\left(\bar{R}_{r}\right)
\end{aligned}
$$

where $A(\kappa)=I_{1}(\kappa) / I_{0}(\kappa)$ and $I_{1}(\kappa)=I_{0}^{\prime}(\kappa)$. In the following, for numerical computation, the approximation $A^{-1}\left(\bar{R}_{r}\right) \approx\left(1.28-0.53 \bar{R}_{r}{ }^{2}\right) \tan \left(\pi \bar{R}_{r} / 2\right)$ proposed in ${ }^{30}$ (with maximum relative error of 0.032 ) will be used. 
When replacing $\beta_{r}$ by its ML estimates $\widehat{\beta}_{r}=\left(\widehat{\mu}_{r}, \widehat{\kappa}_{r}\right)$ in the log-likelihood of eq. 9 the following generalized log-likelihood $\mathcal{L}_{\theta}\left(\Omega_{r} \mid \widehat{\beta}_{r}\right)$ is obtained:

$$
\mathcal{L}\left(\Omega_{r} \mid \widehat{\beta}_{r}\right)=N_{r}\left(\log 2 \pi+\widehat{\kappa}_{r} \bar{R}_{r}-\log I_{0}\left(\widehat{\kappa}_{r}\right)\right)
$$

The MDL criterion associated to a given partition thus contains neither unknown PDF parameter, nor tuning parameter to balance between its different terms. It is then required to find the partition which minimizes this criterion. Following the optimization process proposed in, $\frac{18}{,}$ starting from an initial regular thin grid delimiting $8 \times 8$ pixel regions (as shown in fig. 6 $\mathrm{c}$ and $7 \mathrm{c}$ ), the algorithm consists in alternatively combining three grid modification steps in order to minimize the criterion: a region merging (RegMerg) step in which neighboring regions are merged (allowing the estimation of the number of regions), a node moving (NodMov) step in order to estimate the contour location, and a node removal (NodRem) step in which nodes are progressively removed one by one (allowing to estimate the number of nodes and thus to regularize the contour). Typically, three cycles of RegMerg, NodMov and NodRem steps are sufficient to obtain satisfactory results (sec ${ }^{18}$ for details on the optimization process). For each modification of the grid tested by the optimization algorithm, the parameters $\widehat{\mu}_{r}$ and $\widehat{\kappa}_{r}$ must be re-estimated in order to determine wether or not this modification leads to a decrease of the MDL criterion. Most of the time consuming of the algorithm is thus due to the computation of the $2 \mathrm{D}$ summations over the regions of $\bar{C}_{r}$ and $\bar{S}_{r}$ envolved in the calculation of $\widehat{\mu}_{r}$ and $\widehat{\kappa}_{r}$ (see eq. 10 11 and 12). In order to obtain a fast partitioning algorithm, the implementation proposed in ${ }^{31}$ has been used, allowing one to replace these $2 \mathrm{D}$ summations by 1D summations along the contours of the regions. Using such a technique, the partitioning of a $256 \times 256$ pixel image can then be obtained in less than one second on a standard personal computer (using C programming on a $3.2 \mathrm{GHz}$ processor $\mathrm{PC}$ under Linux).

\section{ORIENTATION ESTIMATION}

\subsection{First results on synthetic images}

When applying this segmentation technique on the noisy orientation image of fig. 1 $\mathrm{b}$ (obtained by applying a $3 \times 3$ GOP estimator), the segmentation results shown on figures $1 \mathrm{e}, \mathrm{f}$ are obtained. It is then proposed to directly estimate the orientation inside a region $\Omega_{r}$ using the whole pixels of this region, i.e. using as large homogeneous samples as possible. The estimation of the orientation is then straightforward as the estimation of the mean orientation inside a region $\Omega_{r}$ is $\bar{\theta}_{r}=\bar{\alpha}_{r} / 2$. The orientation estimated inside each region obtained by the segmentation are shown on fig. 1 g. It appears that these results are better than those obtained using sliding windows, not only in term of the precision of the contours, but also in term of quality since the MAD obtained 

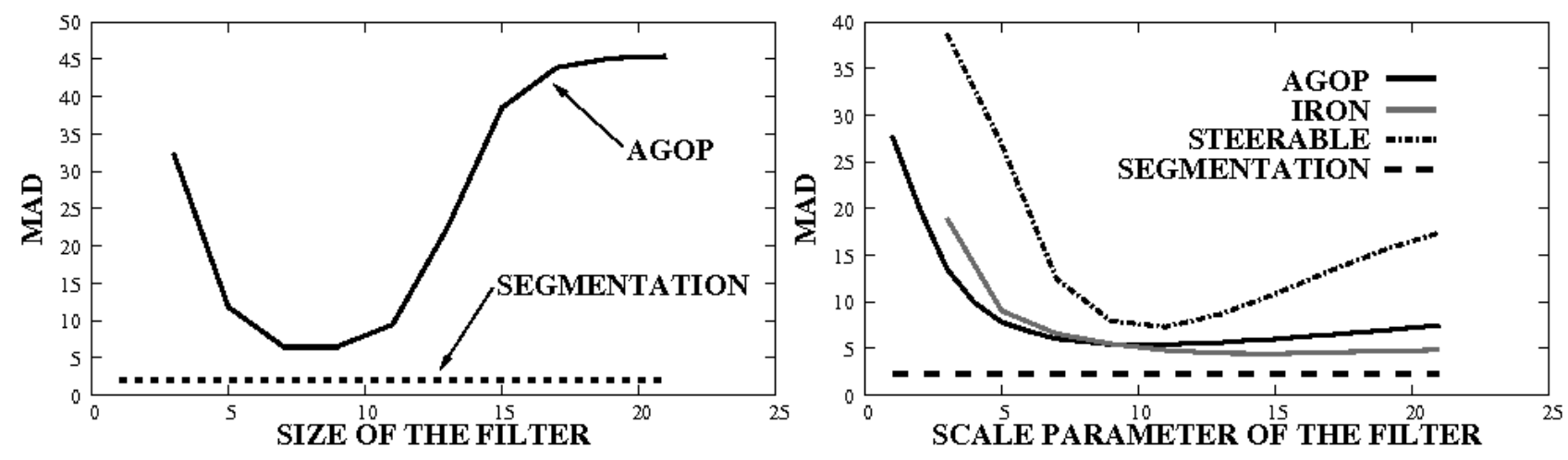

Figure 3. Left: MAD value as a function of the square size $d$ of the sliding windows, in the case of the texture of fig. 1 a. Right: MAD value as a function of the scale parameter for various orientation operators applied to the texture of fig. 4 a. The MAD values obtained using the segmentation-based estimation method have also been reported on these graphs.

using the proposed approach is lower than those obtained with sliding windows as shown on the left part of fig. 3.

\subsection{Comparison experiments}

The method introduced in this paper was shown in the case of fig. 1 a to outperform the AGOP gradient-based orientation estimation by local sliding window averaging. However, it still has not been compared to other standard methods for orientation estimation. In the right part of fig. 3 we plot the evolution of MAD value as a function of scale for various orientation estimation methods. One of them is the steerable filter $\mathrm{E} 2^{232}$ with variable size. The second method is the IRON operator $\frac{17}{17}$ whose size can also be tuned. These methods and the AGOP method discussed above are compared to the proposed segmentation-based method. The textured image used for this experiment is the synthetic image of fig. 4a, which is made of several patches with different pattern frequencies and a noise level $\sigma=50$.

In this case, orientation estimation based on segmentation leads to lower MAD than the other tested methods, whatever the scale, i.e. filter size, of the chosen operator. The corresponding results obtained with the segmentation based method and with each of the other tested methods (when selecting a posteriori the filter size minimizing the MAD) are presented in fig. 4 .

However, at very high noise level, some frontiers between adjacent regions could be missed with the proposed segmentation technique, which can lead to a degradation of the precision of the estimated orientation in these regions. To illustrate this problem, the experiment of fig. 4 has been reproduced on fig. 5 with progressively increasing the standard deviation $\sigma$. Fig. 5 shows the evolution of the MAD as a function of $\sigma$ for two estimation methods: the proposed segmentation based estimation and the best AGOP filter (i.e. the AGOP filter which 


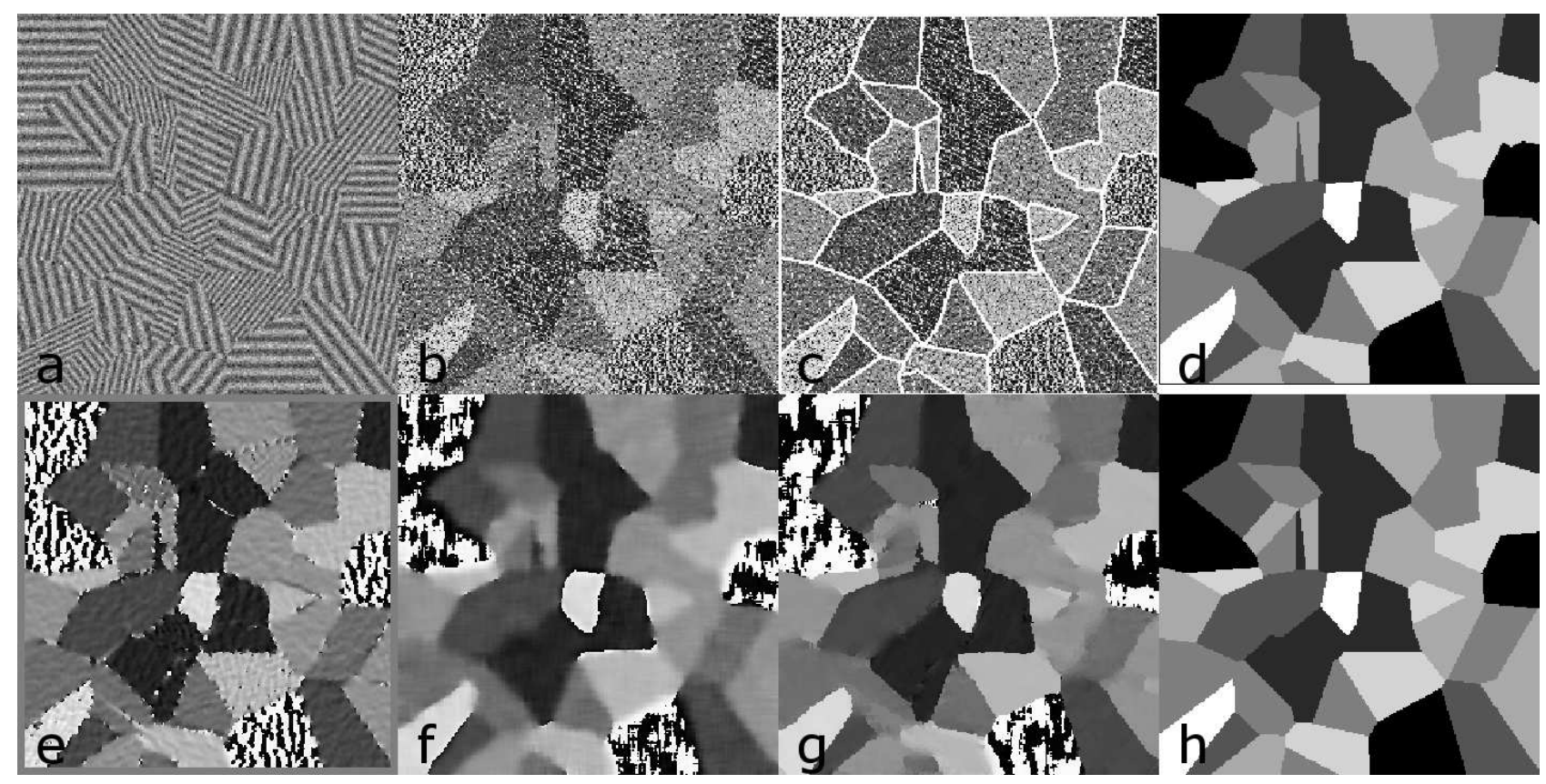

Figure 4. Comparison experiment: (a) Synthetic patchy image in the range $0-100$ with additive noise of standard deviation $\sigma=50$. (b) Orientation estimated using a $3 \times 3$ GOP estimator. (c) Segmentation into homogeneous regions obtained with the proposed technique. (d) Orientation deduced from (c). (e) Best result obtained with the E2 steerable filter (size 11). (f) Best result obtained with the AGOP method (averaging on a $11 \times 11$ window). (g) Best result obtained with the IRON operator (size 15). (h) True orientation on each patch.

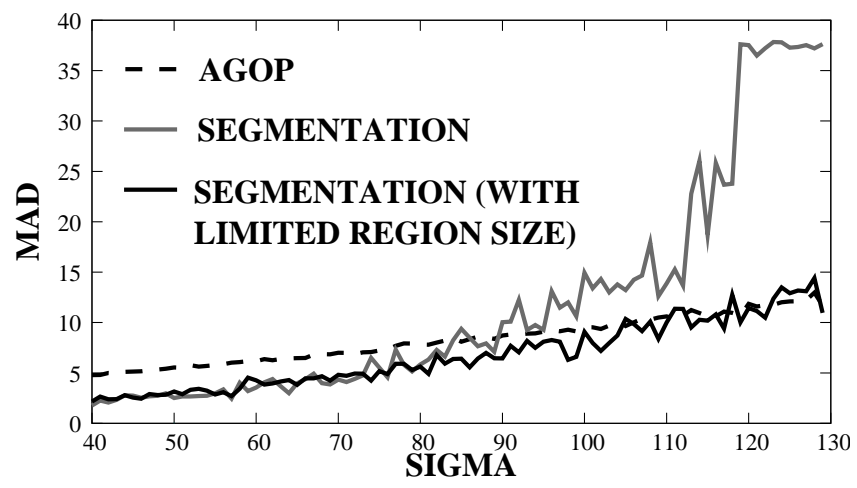

Figure 5. Variation of the MAD when the standard deviation $\sigma$ of the noise added on the image of fig. 4 a increases. The orientation is estimated using the best AGOP filter (dashed black line) and the proposed segmentation based approach, either when no constraint on the region size is imposed (gray solid line) or when limiting the size of the regions that can be merged in the segmentation algorithm (black solid line). 
minimizes the MAD). As soon as $\sigma>90$, the performances of the proposed segmentation based estimation technique rapidly decreases and are outperformed by the best AGOP filter.

Nevertheless, the robustness of this segmentation based technique can be increased at high noise level by limiting the size of the regions in the segmentation step. Indeed, as soon as the regions contain enough pixels to obtain an accurate estimation of the orientation inside the region, increasing the size of the region is not useful anymore, notably since it can lead to the merging of poorly contrasted regions. Since the size of the AGOP filter has been limited to $21 \times 21$ pixels, it has been chosen to impose the same constraint in the segmentation algorithm. For that purpose, in the region merging step of the segmentation algorithm, the merging of two regions with sizes greater than $21 \times 21$ pixels (for both regions) is not allowed. The obtained results are reported on fig. 5. adding such a constraint on the maximal size of the regions allows one to obtain equivalent performances as the segmentation based approach for noise level $\sigma<80$, while keeping at least the performances of the AGOP technique for high noise levels.

\subsection{Application}

To illustrate the use of our approach, the algorithm is finally applied to the processing of microscopy images provided by Snecma Moteurs (Safran group) of a Titane base alloy shown in figures 6 and 7 The structures of interest in such images are the large central regions composed of needles of similar orientation. The objective is to compute needle orientation statistics inside these large regions while getting their contours. The results provided in fig. 6 and 7 show that the large regions are relevantly segmented and that their orientation can be successfully retrieved.

\section{CONCLUSION}

In this paper, a new segmentation based parametric approach for the estimation of orientation in textures has been proposed. This approach relies on an unsupervised MDL based segmentation technique that has been generalized to the case of $\pi$-periodic circular data modeled with Von Mises pdf. Local orientations are computed on each image pixel using a derivative approach. Then pixels are mapped into regions by the segmentation algorithm. Pixels are then assigned the mean orientation of the region they belong to.

Through a series of experiments using synthetic data, the approach appears to process the data successfully in most cases. By maximizing the size of the regions where orientation averaging takes place, accurate orientation inside the regions are estimated and precise boundaries between regions are also determined. On the different images analyzed in this paper, this approach allows one to obtain lower MAD than with the standard orientation estimation techniques that have been tested. Among the various perspectives, a validation of this technique on 


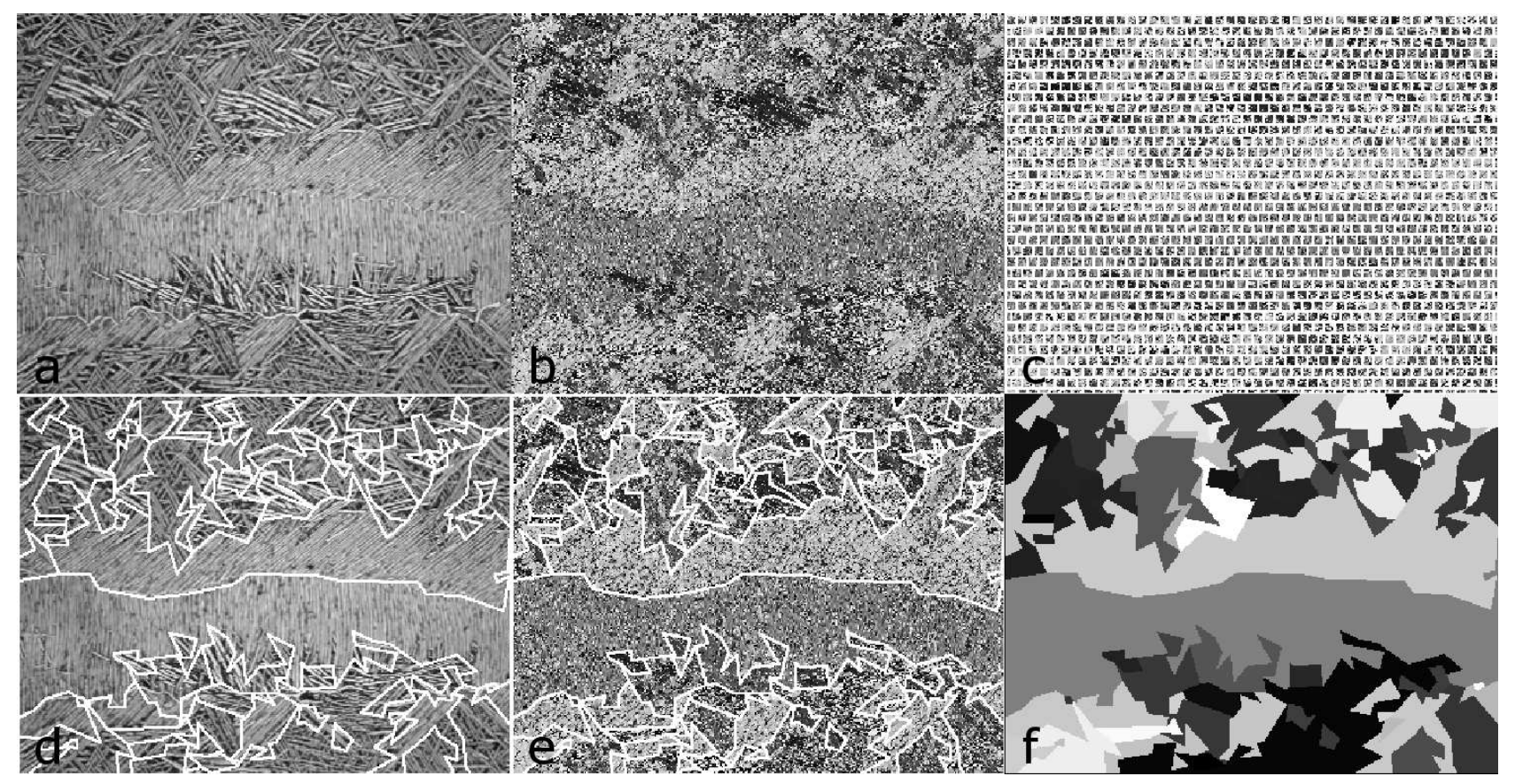

Figure 6. (a) Microscopy image of a Titane base alloy $(360 \times 277$ pixels $)$ provided by Snecma Moteurs (Safran group). (b) Orientation estimated using a $3 \times 3$ GOP operator. (c) Initial grid delimiting $8 \times 8$ pixel regions used to initialize the segmentation algorithm. (d,e) Segmentation results obtained with the proposed approach (either displayed on (a) or (b)) - computational time: $1.4 \mathrm{~s}$. (f) Corresponding orientations estimated inside each regions.

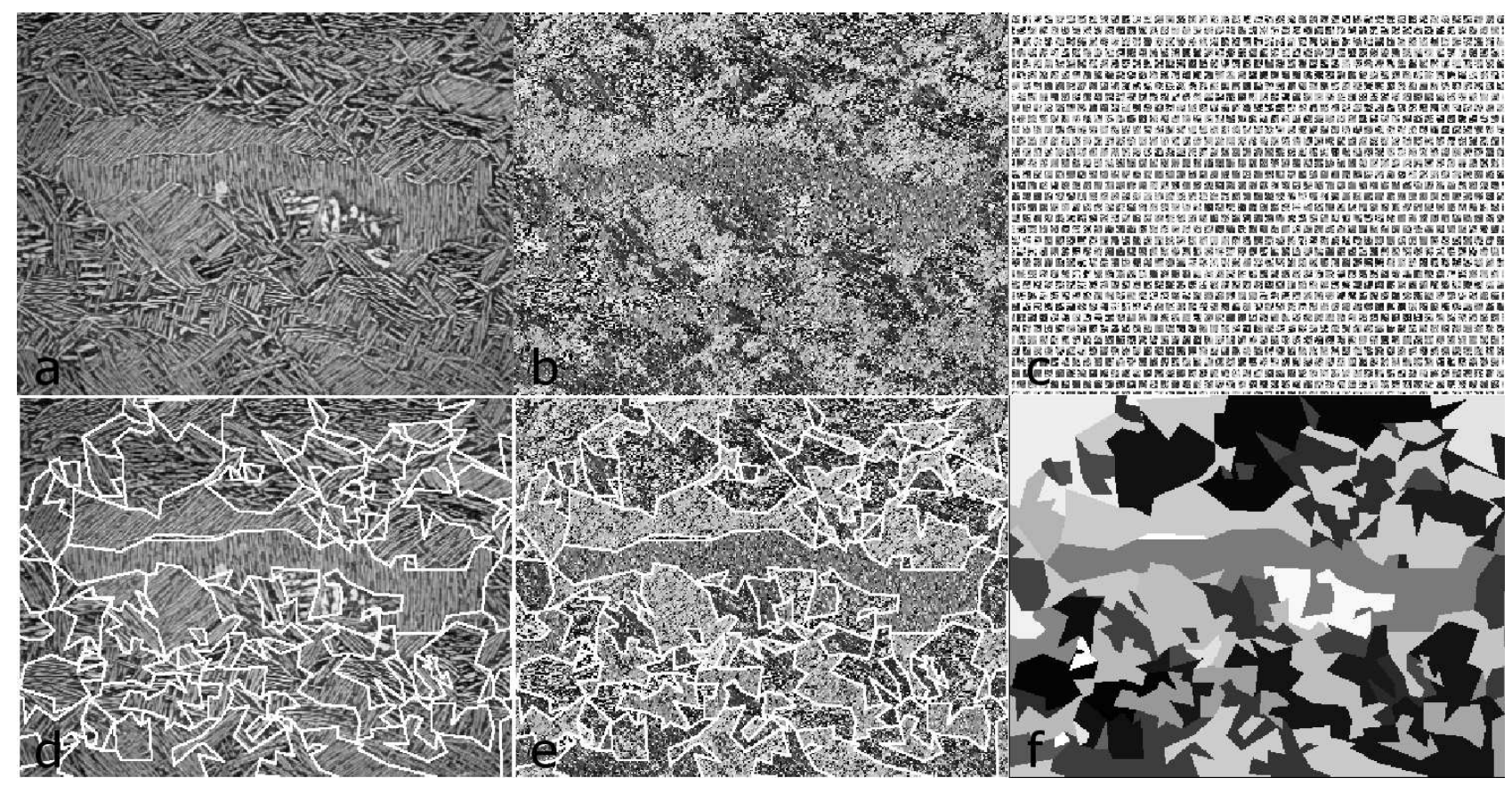

Figure 7. Same as fig. 6 but on another Titane base alloy microscopy image provided by Snecma Moteurs (Safran group). 
other directional textures is possible. Remote sensed images of agricultural scenes (vineyards, row crops, planted forests, etc.), 3D tomographic images of fiber reinforcements in composite materials, or multidirectional textures are such examples.

\section{ACKNOWLEDGMENTS}

The authors would like to thank Snecma Moteurs (Safran group), for providing images and the GdR ISIS group of the French National Research Agency (CNRS) for financial support.

\section{REFERENCES}

[1] Julesz, B. and Bergen, J., "Textons, the fundamentals elements in preattentive vision and perception of textures," The Bell System Technical Journal 62(6), 1619-1645 (1983).

[2] Rao, A. and Lohse, G., "Identifying high level features of texture perception," CVGIP: Graphical Models and Image Processing 55(3), 218-233 (1993).

[3] Kass, M. and Witkin, A., "Analysing oriented patterns," CVGIP: Graphical Models and Image Processing 37, 362-385 (1987).

[4] Chetverikov, D., "Texture analysis using feature-based interaction maps," Pattern Recognition 32, 487-502 (1999).

[5] Germain, C., Da Costa, J.-P., Lavialle, O., and Baylou, P., "Multiscale estimation of vector field anisotropy - application to texture characterization," Signal Processing (83), 1487-1503 (2003).

[6] Bigün, J. and du Buf, J., "N-folded symmetries by complex moments in gabor space and their application to unsupervised texture segmentation," IEEE Transactions on Pattern Analysis and Machine Intelligence 16(1), 80-87 (1994).

[7] Rousson, M., Brox, T., and Deriche, R., "Active unsupervised texture segmentation on a diffusion based feature space," in [Proceedings of the IEEE Conference on Computer Vision and Pattern Recognition (CVPR)], 218-233 (June 2003).

[8] de Luis-García, R., Deriche, R., Rousson, M., and Alberola-López, C., "Tensor processing for texture and colour segmentation," in [Proceedings of the 14th Scandinavian Conference on Image Analysis, volume 3540 of Lecture Notes in Computer Science], 1117-1127 (June 2005).

[9] Knutsson, H., "Representing local structure using tensors," in [Proceedings of the Scandinavian Conference on Image Analysis], (1989). 
[10] Bigün, J., Granlund, G., and Wiklund, J., "Multidimensional orientation estimation with applications to texture analysis and optical flow," IEEE Transactions on Pattern Analysis and Machine Intelligence 13(8), $775-789$ (1991).

[11] de Luis-García, R., Deriche, R., and Alberola-López, C., "Texture and color segmentation based on the combined use of the structure tensor and the image components," Signal Processing 88 (April 2008).

[12] Da Costa, J., Germain, C., Lavialle, O., and Grenier, G., "Segmentation of high resolution remote sensing images : application to the automatic delineation of vine fields," in [Proceedings of the international conference on Agricultural Engineering], (2004).

[13] Da Costa, J., Michelet, F., Germain, C., Lavialle, O., and Grenier, G., "Delineation of vine parcels by segmentation of high resolution remote sensed images," Precision Agriculture 8(1-2), 95-110 (2007).

[14] Grana, C., Borghesani, D., and Cucchiara, R., "Describing texture directions with von mises distributions," $1-4(2008)$.

[15] Mardia, K. and Jupp, J., [Directional Statistics], John Wiley, England (2000).

[16] Le Pouliquen, F., Da Costa, J., Germain, C., and Baylou, P., "A new adaptive framework for unbiased orientation estimation," Pattern Recognition 38, 2032-2046 (2005).

[17] Michelet, F., Da Costa, J., Lavialle, O., Berthoumieu, Y., Baylou, P., and Germain, C., "Estimating local multiple orientations," Signal Processing 87, 1655-1669 (2007).

[18] Galland, F., Bertaux, N., and Réfrégier, P., "Minimum description length synthetic aperture radar image segmentation," IEEE Transactions on Image Processing 12(9), 995-1006 (2003).

[19] Krieger, G. and Zetzsche, C., "Nonlinear image operators for the evaluation of local intrinsic dimensionality," IEEE Transactions on Image Processing 5(6), 1026-1042 (1999).

[20] Aach, T., Mota, C., Stuke, I., M., M., and Barth, E., "Analysis of superimposed oriented patterns," IEEE Transactions on Image Processing 15(12), 3690-3700 (2006).

[21] Rao, A., [A Taxonomy for Texture Description and Identification], Springer, Berlin (1990).

[22] Bigün, J., Bigün, T., and Nilsson, K., "Recognition by symmetry derivatives and the generalized structure tensor," IEEE Transactions on Pattern Analysis and Machine Intelligence 26(12), 1590-1605 (2004).

[23] Rissanen, J., "Modeling by shortest data description," Automatica 14, 465-471 (1978).

[24] Rissanen, J., [Stochastic Complexity in Statistical Inquiry], vol. 15 of Series in Computer Science, World Scientific, Singapore (1989).

[25] Galland, F., Bertaux, N., and Réfrégier, P., "Multicomponent image segmentation in homogeneous regions by stochastic complexity minimization," Pattern Recognition 38(11), 1926-1936 (2005). 
[26] Jones, M. and Pewsey, A., "A family of symmetric distributions on the circle," Journal of the American Statistical Association 100(472), 1422-1428 (2005).

[27] Péron, M.-C., Da Costa, J.-P., Stitou, Y., Germain, C., and Berthoumieu, Y., "Joint linear-circular stochastic models for texture classification," in [Proceedings of ICASSP], 1073-1076 (2009).

[28] Galland, F., Jaegler, A., Allain, M., Savéry, D., and Réfrégier, P., "Smooth contour coding with minimal description length active grid segmentation techniques," Pattern Recognition Letters 32(5), 721-730 (2011).

[29] Shannon, C. E., "A mathematical theory of communication," Bell Syst. Tech. J. 27, 379-423 ; 623 - 656 (1948).

[30] Dobson, A. J., "Simple approximations for the von mises concentration statistic," Applied Statistics 27(3), 345-347 (1978).

[31] Chesnaud, C., Réfrégier, P., and Boulet, V., "Statistical region snake-based segmentation adapted to different physical noise models," IEEE Trans. Pattern Anal. and Machine Intell. 21, 1145-1157 (nov 1999).

[32] Freeman, W. and Adelson, E., "The design and use of steerable filters," IEEE Transactions on Pattern Analysis and Machine Intelligence 13(9), 891-906 (1991). 


\section{Figure captions}

Figure 1. (a) Textured image obtained by adding to a non noisy image (with grey levels between 0 and 100) a zero-mean Gaussian noise with standard deviation $\sigma=50$. The orientation estimated using a $3 \times 3$ GOP estimator is shown on (b) and the smoothing of this orientation with a $d \times d$ sliding windows is shown for $d=7$ (c) and $d=11$ (d). The segmentation into homogeneous regions obtained with the proposed technique is shown on (e,f) (either displayed on (a) or (b)) and the associated estimated orientations are shown on $(\mathrm{g})$. The orientation ground truth is shown on (h). In this case $\widetilde{d}^{M A D}=7$.

Figure 2. Same as figure 1, but with $\sigma=100$. In this case $\widetilde{d}^{M A D}=11$.

Figure 3. Left: MAD value as a function of the square size $d$ of the sliding windows, in the case of the texture of fig. 1 a. Right: MAD value as a function of the scale parameter for various orientation operators applied to the texture of fig. 4 a. The MAD values obtained using the segmentation-based estimation method have also been reported on these graphs.

Figure 4. Comparison experiment: (a) Synthetic patchy image in the range $0-100$ with additive noise of standard deviation $\sigma=50$. (b) Orientation estimated using a $3 \times 3$ GOP estimator. (c) Segmentation into homogeneous regions obtained with the proposed technique. (d) Orientation deduced from (c). (e) Best result obtained with the E2 steerable filter (size 11). (f) Best result obtained with the AGOP method (averaging on a $11 \times 11$ window). (g) Best result obtained with the IRON operator (size 15). (h) True orientation on each patch.

Figure 5. Variation of the MAD when the standard deviation $\sigma$ of the noise added on the image of fig. 4 a increases. The orientation is estimated using the best AGOP filter (dashed black line) and the proposed segmentation based approach, either when no constraint on the region size is imposed (gray solid line) or when limiting the size of the regions that can be merged in the segmentation algorithm (black solid line).

Figure 6. (a) Microscopy image of a Titane base alloy $(360 \times 277$ pixels $)$ provided by Snecma Moteurs (Safran group). (b) Orientation estimated using a $3 \times 3$ GOP operator. (c) Initial grid delimiting $8 \times 8$ pixel regions used to initialize the segmentation algorithm. (d,e) Segmentation results obtained with the proposed approach (either displayed on (a) or (b)) - computational time: $1.4 \mathrm{~s}$. (f) Corresponding orientations estimated inside each regions.

Figure 7. Same as fig. 6 but on another Titane base alloy microscopy image provided by Snecma Moteurs (Safran group). 